\title{
Short Chain Fatty Acid HQK-1004
}

National Cancer Institute

\section{Source}

National Cancer Institute. Short Chain Fatty Acid HQK-1004. NCI Thesaurus. Code C88290.

A short chain fatty acid (SCFA) with potential herpes simplex virus thymidine kinase gene (HSV-TK)-inducing activity. Upon administration, short chain fatty acid HQK-1004 may induce the expression of thymidine kinase (TK) by a silenced HSV-TK, which may activate a co-administered antiviral prodrug such as ganciclovir, resulting in the destruction of virally-infected cancer cells. 